\title{
Management of acute intracerebral haemorrhage - an update
}

\author{
Authors: Zhe Kang Law, ${ }^{A, B}$ Jason P Appleton, ${ }^{A}$ Philip M Bath ${ }^{C, D}$ and Nikola Sprigg ${ }^{E}$
}

\begin{abstract}
Managing acute intracerebral haemorrhage is a challenging task for physicians. Evidence shows that outcome can be improved with admission to an acute stroke unit and active care, including urgent reversal of anticoagulant effects and, potentially, intensive blood pressure reduction. Nevertheless, many management issues remain controversial, including the use of haemostatic therapy, selection of patients for neurosurgery and neurocritical care, the extent of investigations for underlying causes and the benefit versus risk of restarting antithrombotic therapy after an episode of intracerebral haemorrhage.
\end{abstract}

\section{Introduction}

Spontaneous intracerebral haemorrhage $(\mathrm{sICH})$ is a common medical emergency with a mortality rate of $40 \%$ at 1 month. ${ }^{1}$ Although advancement has been made in the treatment of ischaemic stroke, research into the management of spontaneous intracerebral haemorrhage has lagged behind. Nevertheless, recent evidence provides some insight into the optimal management of patients with sICH, which can improve outcome.

\section{Neuroimaging for sICH}

Apart from diagnosing sICH, the main aims of neuroimaging are to detect complications, such as haematoma expansion (Fig 1), intraventricular extension, hydrocephalus, cerebral oedema and mass effect. In addition, neuroimaging is crucial to identify the underlying aetiology. Urgent computerised tomography (CT) scan and magnetic resonance imaging (MRI) are equally effective in diagnosing sICH. CT angiography is often done in a research setting to look for contrast extravasation or positive spot sign, which predicts haematoma

Authors: ${ }^{\text {A }}$ clinical research fellow and PhD student, University of Nottingham, UK; ${ }^{\text {B }}$ lecturer and neurologist, National University of Malaysia, Kuala Lumpur, Malaysia; ' professor of stroke medicine, University of Nottingham, UK; ${ }^{D}$ honorary consultant physician, Nottingham University Hospitals NHS Trust, Nottingham, UK; ${ }^{E}$ clinical associate professor and consultant stroke physician, University of Nottingham, UK expansion. ${ }^{2} \mathrm{CT}$ angiography-positive spot sign has a sensitivity of $63 \%$ and specificity of $90 \%$ in predicting haematoma expansion. ${ }^{2}$ Predicting haematoma expansion could be useful in stratifying patients at risk of deterioration.

It was previously thought that investigations for an underlying structural or vascular lesion should be reserved for patients aged $<45$ years, with normotension and lobar haemorrhage. ${ }^{3}$ This assumption is unreliable as angiographic studies showed that up to $20 \%$ of patients with sICH have underlying vascular abnormalities; many of these patients were elderly and hypertensive with deep-seated haematomas. ${ }^{4}$ Hence, all patients should be considered for non-invasive vascular imaging (CT angiography or magnetic resonance angiography) and structural imaging (MRI). These should be performed as early as possible to enable early interventions. Invasive digital subtraction angiography should be reserved for patients where the suspicion of vascular anomalies remains high despite negative non-invasive imaging. ${ }^{5}$ Table 1 illustrates the investigations for causes of sICH.

\section{Key points}

All patients should be actively treated in an acute stroke unit or intensive care unit with avoidance of early do not attempt resuscitation orders

In patients presenting within 6 hours of onset, lowering systolic blood pressure to $<140 \mathrm{mmHg}$ within 1 hour of admission is safe and may be beneficial

Patients taking anticoagulation should receive urgent reversal with the appropriate antidote

Patients with superficial haematoma and Glasgow Coma Scale score of 9-12 may benefit from neurosurgery

All patients with spontaneous intracerebral haemorrhage should be considered for investigation of underlying aetiology using structural and vascular neuroimaging

KEYWORDS: Antithrombotic, blood pressure, intracerebral haemorrhage, management, neurosurgery, stroke 

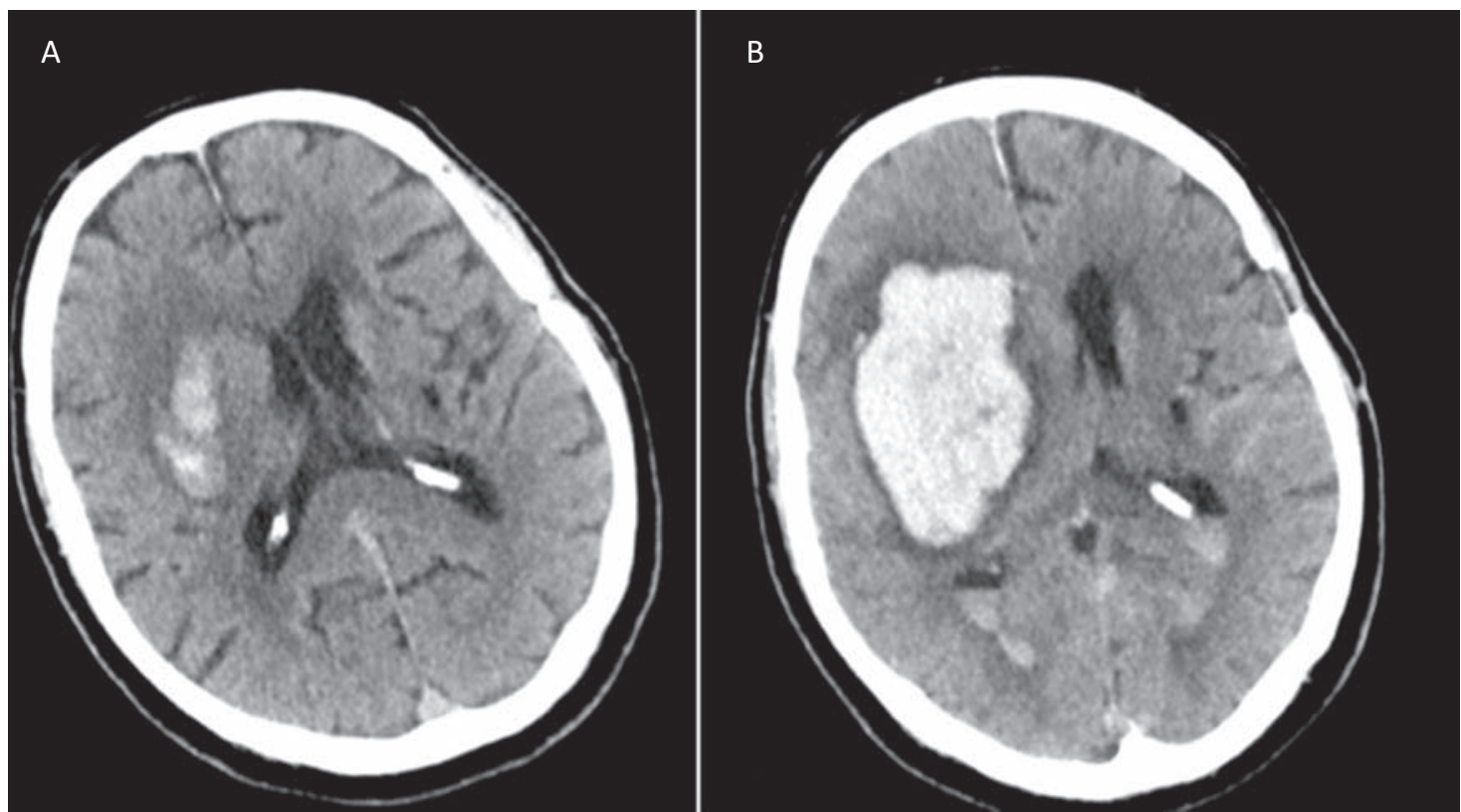

Fig 1. Haematoma expansion. A - non-contrasted CT brain showing right basal ganglia haematoma; B - CT brain 24 hours later showing haematoma expansion with peri-haematomal oedema, midline shift and intraventricular extension. Courtesy of Mark Sampson, University of Nottingham. CT = computerised tomography

Table 1. Causes of spontaneous intracerebral haemorrhage, investigations and characteristics

\begin{tabular}{|c|c|c|}
\hline Causes & Investigations & Characteristics \\
\hline Hypertensive arteriopathy & $\begin{array}{l}\text { MRI including T2 GRE } \\
\text { or SWI sequence }\end{array}$ & $\begin{array}{l}\text { Presence of deep-seated cerebral microbleeds, leukoaraiosis and lacunar infarctions } \\
\text { is supportive. }\end{array}$ \\
\hline $\begin{array}{l}\text { Cerebral amyloid } \\
\text { angiopathy }\end{array}$ & $\begin{array}{l}\text { MRI including T2 GRE } \\
\text { or SWI sequence } \\
\text { Apolipoprotein E } \\
\text { ( } \varepsilon 2 \text { and } \varepsilon 4 \text { alleles) }\end{array}$ & $\begin{array}{l}\text { MRI: lobar haemorrhage, microhaemorrhages at cortico-subcortical junctions. } \\
\text { Carrier of Apolipoprotein } \varepsilon 2 \text { and } \varepsilon 4 \text { alleles have higher risk of recurrent sICH. }\end{array}$ \\
\hline Cavernoma & MRI & $\begin{array}{l}\text { MRI: heterogeneous signal intensities on T1 and T2 with a hypointense } \\
\text { surrounding rim. Typically described as 'popcorn' or 'berry' appearance. }\end{array}$ \\
\hline $\begin{array}{l}\text { Arteriovenous } \\
\text { malformation }\end{array}$ & MRI, MRA, DSA & MRA/DSA: dilated arteries feeding a nidus and multiple draining veins. \\
\hline Intracranial aneurysm & CTA, MRI, MRA, DSA & $\begin{array}{l}\text { Presence of subarachnoid haemorrhage. DSA is the gold standard in detecting } \\
\text { aneurysm. }\end{array}$ \\
\hline $\begin{array}{l}\text { Cerebral venous } \\
\text { thrombosis }\end{array}$ & CTV, MRV & $\begin{array}{l}\text { Clinical signs of increased intracranial pressure. Symmetrical haemorrhagic } \\
\text { infarctions. Filling defect (flow void) in the venous system. }\end{array}$ \\
\hline Dural arteriovenous fistula & CTA, MRA, DSA & Haematoma location near venous sinus and cortical veins. \\
\hline Brain tumour/metastasis & CECT/MRI & $\begin{array}{l}50 \% \text { of patients with brain metastasis will have multiple lesions that may show } \\
\text { contrast enhancement. }\end{array}$ \\
\hline Anticoagulant & $\begin{array}{l}\text { History/coagulation } \\
\text { profile }\end{array}$ & $\begin{array}{l}\text { Prolongation of coagulation profile. In patients on NOACs, the presence of normal } \\
\text { coagulation profile does not indicate absence of coagulopathy. }\end{array}$ \\
\hline Thrombolysis agent & History & $\begin{array}{l}\text { Diagnosis of prior ischaemic stroke or myocardial infarction requiring thrombolysis } \\
\text { is usually evident. }\end{array}$ \\
\hline $\begin{array}{l}\text { Cocaine and } \\
\text { sympathomimetic drugs }\end{array}$ & $\begin{array}{l}\text { History } \\
\text { Urine cocaine levels }\end{array}$ & Elevated urine cocaine levels can be detected within 72 hours of cocaine use. \\
\hline
\end{tabular}




\section{Acute medical management}

The benefits of the acute stroke unit are well established. Admission to an acute stroke unit improves outcome in terms of death and dependency (Odds ratio 0.79, 95\% confidence interval (CI) $0.68-0.90 ; \mathrm{p}=0.0007) .{ }^{6}$ Neurological complications of sICH - including haematoma expansion, peri-haematomal oedema, intraventricular extension of haemorrhage, hydrocephalus, seizures and non-neurological complications, such as aspiration pneumonia and venous thromboembolism - all lead to poor prognosis. ${ }^{7,8}$ Therefore, the aim of acute management is to detect and prevent these complications. Importantly, an early do not attempt resuscitation (DNAR) order should be avoided in the first few days since it limits optimal medical care and results in poor outcome. ${ }^{9,10}$ Table 2 summarises the recommendations in management of sICH.

\section{Blood pressure control}

Very elevated blood pressure (BP) increases the risk of haematoma expansion, leading to a poor prognosis in sICH. ${ }^{11}$ Conversely, there is a concern that lowering BP acutely reduces cerebral perfusion pressure and leads to peri-haematomal ischaemia and infarction. However, recent trials have demonstrated the safety of intensive BP reduction. INTERACT-2 (Intensive Blood Pressure Reduction in Acute Cerebral Hemorrhage Trial 2), involving 2,839 patients, showed that those with intensive BP reduction did not have an increased rate of ischaemic events. ${ }^{12}$ Similarly, the ATACH
(Antihypertensive Treatment of Acute Cerebral Hemorrhage) study demonstrated safety of intensive blood pressure reduction in sICH. ${ }^{13}$ Additionally, a subgroup analysis of ENOS (Efficacy of Nitric Oxide in Stroke) trial patients with sICH presenting within 6 hours showed benefit of BP reduction with glyceryl trinitrate on functional outcome..$^{14}$ A meta-analysis showed that intensive blood pressure reduction is safe, with a non-significant tendency towards a favourable outcome, which may be mediated through attenuation of haematoma expansion. ${ }^{15}$ Recent guidelines suggest lowering BP to a systolic of $140 \mathrm{mmHg}$ within 1 hour in those presenting within 6 hours of symptom onset is safe and may have a beneficial effect on functional outcome. ${ }^{16-18}$

The most recent development of $\mathrm{BP}$ treatment in $\mathrm{sICH}$ is the ATACH-2 trial, which was stopped early in view of futility. Although there was no significant difference in death or dependency between the intensive and guideline treatment groups, there was a non-significant reduction in haematoma expansion in the intensive treatment arm. In addition, there were higher renal adverse events in those treated with intensive BP reduction. ${ }^{19}$ There are several potential reasons why ATACH-2 had different findings compared to previous trials. Patients in the trial received intravenous antihypertensive medication pre-randomisation to ensure compliance with guidelines. Further, the BP reduction was much larger than seen in previous trials - from a baseline systolic BP (SBP) of approximately $200 \mathrm{mmHg}$, the intensive arm achieved a mean minimum SBP of $129 \mathrm{mmHg}$, while the standard treatment arm attained a mean minimum SBP of $141 \mathrm{mmHg}$.

Table 2. General management of patients with spontaneous intracerebral haemorrhage

\begin{tabular}{|c|c|}
\hline Section & Recommendations \\
\hline $\begin{array}{l}\text { General care/nursing } \\
\text { care }\end{array}$ & $\begin{array}{l}\text { All patients should be admitted to an acute stroke unit or intensive care unit. Frequent vital sign checks, } \\
\text { neurological assessment and continuous cardiopulmonary monitoring (pulse oximetry, automated BP cuff and } \\
\text { ECG telemetry) should be performed. }\end{array}$ \\
\hline Airway protection & $\begin{array}{l}\text { Ventilation should be considered for patients with impending ventilator failure, at risk of aspiration, signs of } \\
\text { raised intracranial pressure or brainstem dysfunction. }\end{array}$ \\
\hline \multirow[t]{2}{*}{ ICP monitoring } & $\begin{array}{l}\text { ICP should be monitored in patients with IVH, hydrocephalus, signs of transtentorial herniation or GCS } \leq 8 \text { that } \\
\text { were presumably caused by haematoma mass effect. }\end{array}$ \\
\hline & Maintenance of ICP of $<20 \mathrm{mmHg}$ and CPP of $50-70 \mathrm{mmHg}$ is reasonable. \\
\hline Blood pressure & $\begin{array}{l}\text { In patients with acute sICH presenting within } 6 \text { hours of symptom onset, blood pressure should be lowered to } \\
\text { SBP }<140 \mathrm{mmHg} \text { within } 1 \text { hour. }\end{array}$ \\
\hline VTE prophylaxis & Intermittent pneumatic compression should be used. Graduated compression stockings should be avoided. \\
\hline Dysphagia screening & Patients should be kept nil by mouth until a formal swallowing assessment is done. \\
\hline Fever & High grade fever of $>38^{\circ} \mathrm{C}$ should be treated with paracetamol and/or tepid sponging. \\
\hline Glucose & Hypoglycaemia and hyperglycaemia should be treated. In treating hyperglycaemia, insulin should be used. \\
\hline Intravenous fluid & Avoid over-hydration. Avoid dextrose-containing fluid. \\
\hline \multirow[t]{2}{*}{ Seizure } & $\begin{array}{l}\text { Patients with clinical seizure(s) should be treated with antiepileptic agent(s). Patients without seizure should } \\
\text { not be prophylactically treated with antiepileptic agent(s). }\end{array}$ \\
\hline & $\begin{array}{l}\text { Patients with altered conscious level and epileptic discharges detected on electroencephalography should be } \\
\text { treated with antiepileptic agents. }\end{array}$ \\
\hline DNAR order & Early DNAR order should be avoided in the first few days as it may limit active medical treatment. \\
\hline
\end{tabular}

$\mathrm{BP}=$ blood pressure; $\mathrm{CPP}=$ cerebral perfusion pressure; $\mathrm{DNAR}=$ do not attempt resuscitation; $\mathrm{ECG}=$ electrocardiogram; $\mathrm{GCS}=$ Glasgow Coma Scale; ICP = intracranial pressure; IVH = intraventricular haemorrhage; $\mathrm{SBP}=$ systolic blood pressure; $\mathrm{SICH}=$ spontaneous intracerebral haemorrhage; VTE = venous thromboembolism 
In contrast, the baseline $\mathrm{BP}$ was approximately $179 \mathrm{mmHg}$ in INTERACT-2, with the intensive and standard treatment groups achieving a SBP of $150 \mathrm{mmHg}$ and $164 \mathrm{mmHg}$ at 1 hour post randomisation, respectively. These results suggest that the degree of BP reduction in the standard arm of ATACH-2 is similar to the intensive arm of INTERACT-2. In addition, all patients in ATACH-2 were treated with intravenous nicardipine, which has a weak antiplatelet effect, possibly negating the benefit achieved by BP reduction.

In conclusion, a target SBP of $140 \mathrm{mmHg}$ is probably safe, while more intensive lowering of SBP to $120 \mathrm{mmHg}$ does not appear to add further benefit. Secondary prevention of stroke with $\mathrm{BP}$ control is crucial but is beyond the scope of this article. $^{20}$

\section{Haemostatic therapy}

The aim of haemostatic therapy is to prevent continued bleeding of the haematoma. The clinical approach depends on whether the patient has received proximal anticoagulation therapy; such patients have worse outcomes in part due to continued bleeding, thus warranting urgent reversal.

\section{Vitamin $\mathrm{K}$ antagonist-related ICH}

Vitamin K antagonists (VKA), such as warfarin, should be withheld and intravenous vitamin $\mathrm{K} 10 \mathrm{mg}$ given immediately. Prothrombin complex concentrate (PCC) normalises INR more rapidly than fresh frozen plasma (FFP) and this should be given as soon as possible. In the $2016 \mathrm{INCH}$ (intracranial haemorrhage related to vitamin $\mathrm{K}$ antagonists) trial, 4 -factor PCC reversed INR to $<1.2$ within 3 hours in $67 \%$ (18 of 27 patients) compared with FFP ( $9 \%, 2$ of 23 patients) in sICH. ${ }^{21}$ Protocols should be in place to reduce delay in giving PCC, including implementing point-of-care INR measurement and storage of PCC in emergency departments or hyperacute stroke units. $^{22}$

\section{Novel oral anticoagulant-related ICH}

Novel oral anticoagulants (NOACs), including dabigatran, rivaroxaban, apixaban and edoxaban, are widely used as an alternative to VKA. Idarucizumab, a reversal agent specific to dabigatran, is effective within minutes of administration and should be used in all cases of dabigatran-related sICH. ${ }^{23}$ Andexanet, a reversal agent for rivaroxaban, apixaban and edoxaban is not yet available. ${ }^{24}$ Where an effective antidote is not available, NOACs-related sICH can be treated supportively with PCC, FFP or tranexamic acid. ${ }^{25}$

\section{Anti-platelet-related ICH}

Despite plausible physiological benefits of platelet transfusion in reducing the extent of $\mathrm{ICH}$ in patients taking anti-platelet agents, the PATCH (Platelet transfusion versus standard care after acute stroke due to spontaneous cerebral haemorrhage associated with anti-platelet therapy) trial reported that giving platelet transfusions was in fact harmful and should be avoided. ${ }^{26}$ Among 97 patients who were randomised to receive platelet transfusion and 93 patients who received standard care, death and dependency was significantly higher in patients who received platelet transfusion (adjusted common odds ratio $2.05,95 \%$ CI $1.18-3.56$; $\mathrm{p}=0.0114)$. Forty patients $(42 \%)$ who received platelet transfusion had serious adverse events compared with 28 patients (29\%) who received standard care. $^{26}$

\section{Non-antithrombotic related ICH}

There is currently no haemostatic agent that is proven to limit haematoma expansion in non-anticoagulant related $\mathrm{ICH} .{ }^{27}$ The most widely researched agent, activated recombinant factor VIIa, did not improve clinical outcome or reduce haematoma size. ${ }^{28}$ Ongoing trials are investigating efficacy of tranexamic acid in $\mathrm{sICH}^{29,30}$

\section{Role of neurosurgery}

Not all patients with sICH benefit from surgery. Several factors should be taken into consideration when deciding on surgery, including haematoma location, patient's prognostic factors, timing and type of surgery.

\section{Cerebellar haemorrhage}

Cerebellar haemorrhage may cause compression of the fourth ventricle (resulting in obstructive hydrocephalus) and of the brainstem leading to herniation syndrome. Therefore, posterior decompression should be performed in patients with cerebellar haematoma of $>3 \mathrm{~cm}$ and those that result in hydrocephalus or brainstem compression. ${ }^{31}$ Insertion of an intraventricular drain or ventriculostomy without decompression is insufficient. ${ }^{17}$

\section{Supratentorial haemorrhage}

There is no evidence that routine surgery for all patients with supratentorial haemorrhage is beneficial. In the STICH (Surgical Treatment for Ischemic Heart Failure) trial, involving 1,033 patients with sICH, early surgery did not improve outcome. ${ }^{32}$ However, there was apparent benefit in patients and those with superficial lobar haematoma. The subsequent STICH II (Surgical Trial in Lobar Intracerebral Hemorrhage) trial reported that early surgery non-significantly reduced mortality in patients with superficial lobar haematoma $(<1 \mathrm{~cm}$ from surface) without intraventricular extension and Glasgow Coma Scale (GCS) score of $>8 .{ }^{33}$ A meta-analysis including STICH II and 14 other trials concluded overall benefit for early surgery although this should be interpreted with caution because of significant heterogeneity between the trials. ${ }^{33}$

\section{Timing of surgery}

Surgery performed within 8 hours from onset appears to be beneficial. ${ }^{34}$ However, ultra-early surgery performed within 4 hours from onset is not beneficial and might be harmful. ${ }^{35}$

\section{Type of surgery}

Craniotomy and evacuation of haematoma was the procedure most commonly performed on patients in large clinical trials. The decision to undertake craniotomy and evacuation of 
haematoma on a patient with supratentorial haemorrhage should be individualised with consideration of the following points:

$>$ patients with superficial lobar haemorrhage $<1 \mathrm{~cm}$ from surface may benefit, whereas patients with deep seated haemorrhage do not appear to benefit

$>$ evacuation of haematoma should be considered for patients with intermediate GCS of 9-12; patients with poor GCS of $<8$ or fully alert are unlikely to benefit.

On the other hand, decompressive craniectomy may be lifesaving in patients with coma, large haematoma with midline shift and medically intractable raised intracranial pressure based on case-control studies and case series. ${ }^{17,36}$ In addition, when sICH is complicated with hydrocephalus, the placement of an external ventricular drain lowers intracranial pressure and may reduce mortality. ${ }^{17,37}$ An external ventricular drain allows for monitoring of intracranial pressure.

Phase III studies testing minimally invasive surgery and intraventricular thrombolysis for intraventricular haemorrhage are ongoing and may change our approach in the near future. $^{38,39}$

\section{Restarting antithrombotic therapy}

In all cases of sICH (except if secondary to cerebral venous thrombosis, where anticoagulation should be instigated), antiplatelet therapy and anticoagulants should be withheld in the acute setting. ${ }^{40}$ Thereafter, it is unclear whether and when antithrombotic therapy should be restarted. The decision to restart should be tailored to the individual after carefully considering the benefits of antithrombotic therapy against the risks of recurrent sICH.

\section{Risk of recurrent ICH}

Most rebleeding in sICH occurs in the first 24 hours. Subsequently, the annual risk of recurrent $\mathrm{sICH}$ is estimated to be $2-3 \% .{ }^{41}$ However, certain factors are associated with higher risk of recurrent $\mathrm{sICH}$, including poorly controlled hypertension (three- to fourfold risk), ${ }^{42}$ cerebral amyloid angiopathy (Fig 2, up to $22 \%$ per year), ${ }^{43}$ lobar haemorrhage (3-14\% per year), ${ }^{44}$ arteriovenous malformation (18\% per year) and cerebral microbleeds on MRI (eightfold risk). ${ }^{45}$ The risk of recurrent sICH increases three- to fivefold in patients taking VKA. ${ }^{46}$ In addition, VKA is associated with larger haematomas and worse outcome. NOACs have been considered to have lower risk of recurrent sICH compared with VKA (relative risk of 0.46). ${ }^{47}$ As for antiplatelet therapy, there is conflicting evidence to whether it increases the risk of recurrent sICH. Some studies concluded that antiplatelet therapy increased the risk of recurrent sICH while others did not. ${ }^{43}$

\section{Risk of thromboembolism and protective effect of antithrombotic therapy}

Prosthetic heart valves are highly thrombogenic and carry a $4-8 \%$ annual risk of thromboembolism if not anticoagulated. ${ }^{48}$ VKA reduces the risk of thromboembolism by $75 \% .{ }^{49}$ NOACs carry a lower risk of bleeding than VKA but cannot be used in patients with prosthetic valves. A trial evaluating the use of dabigatran in prosthetic valves was terminated early due

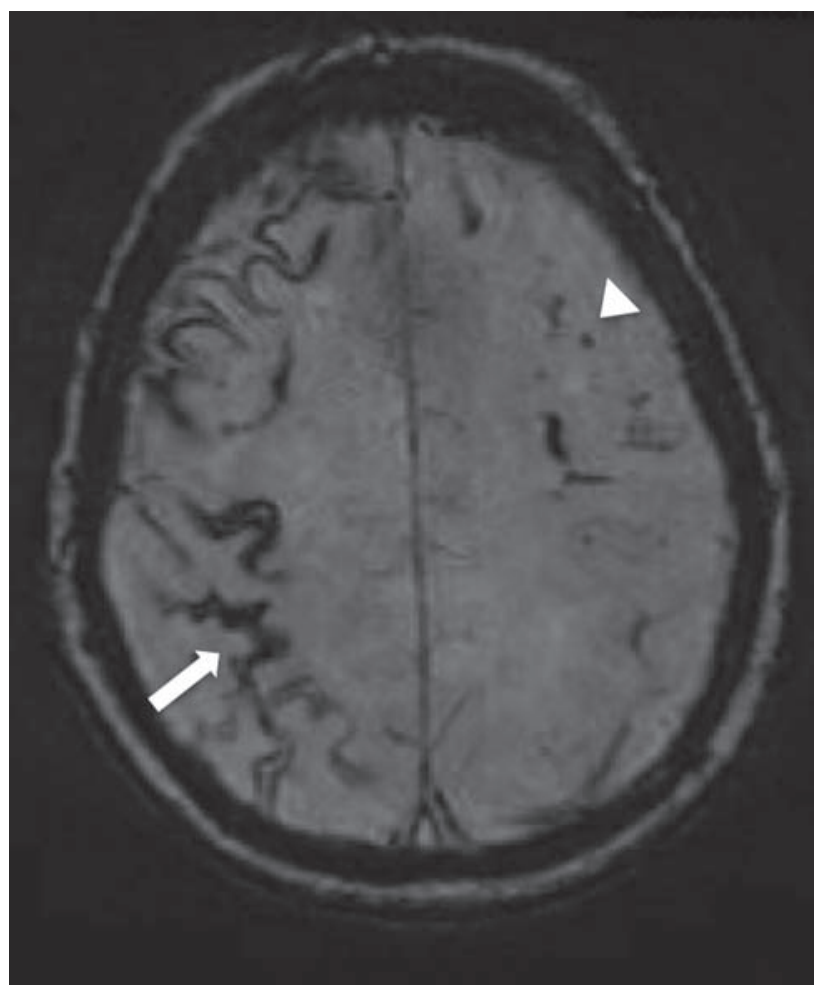

Fig 2. Cerebral amyloid angiopathy. Magnetic resonance susceptibilityweighted imaging sequence showing superficial siderosis (white arrow) and cerebral microhaemorrhage (white arrow head), which are features supportive of a diagnosis of cerebral amyloid angiopathy.

to excessive adverse events. ${ }^{50}$ The annual risk of systemic thromboembolism in patients with atrial fibrillation varies between $1 \%$ and $15 \%$, depending on $\mathrm{CHA}_{2} \mathrm{DS}_{2}$-VASc score. ${ }^{51}$ The risk ranges from nearly $0 \%$ when $\mathrm{CHA}_{2} \mathrm{DS}_{2}$-VASc score is 0 to $15 \%$ when the score is 9 . Treatment with anticoagulation confers a $64 \%$ relative risk reduction of stroke. ${ }^{52}$

There are no randomised controlled trials to guide decision making in restarting antithrombotic therapy in patients with prior sICH. One observational study of 1,752 patients with atrial fibrillation and $\mathrm{ICH}$ suggested an overall mortality benefit of restarting oral anticoagulation compared with antiplatelet therapy or no antithrombotics. ${ }^{53}$ However, the study did not take into account the location of $\mathrm{ICH}$, presence of microbleeds and other risk factors for recurrent ICH. Based on a decision analysis model, Eckman et al suggested that anticoagulation should be avoided in all cases of lobar haemorrhage, but could be offered to deep haemorrhage if the risk of thrombotic events is $>7 \%$ per year. ${ }^{54}$ However, it is uncertain whether antiplatelet therapy should be offered when anticoagulation is deemed not suitable. In the face of clinical equipoise, enrolment into a clinical trial should be considered. One such trial in the UK is RESTART (REstart or STop Antithrombotics Randomised Trial), which is exploring the risk of recurrent $\mathrm{sICH}$ in patients who are given antiplatelet therapy. ${ }^{55}$ Where this is not possible, the following points can be considered:

> In patients with high risk of thromboembolism (prosthetic heart valves, high-risk atrial fibrillation, high-risk coronary 
artery disease) and low risk of recurrent sICH (deep haemorrhage, well controlled blood pressure) antithrombotic therapy should be considered.

$>$ Conversely, antithrombotics are discouraged in patients with high risk of recurrent sICH (cerebral amyloid angiopathy, presence of multiple microbleeds, lobar haemorrhage, uncontrolled hypertension).

$>$ For patients with high-risk non-valvular atrial fibrillation and a high risk of bleeding, percutaneous left atrial appendage occlusion may be considered for stroke prevention. Left atrial appendage occlusion is non-inferior to warfarin for stroke prevention in atrial fibrillation based on observational data and the PROTECT AF (Watchman Left Atrial Appendage System for Embolic Protection in Patients With Atrial Fibrillation) trial. ${ }^{56,57}$

> While NOACs have a lower bleeding risk than VKAs, there is insufficient evidence to recommend using NOACs instead of VKAs after anticoagulation-related sICH. An ongoing trial, APACHE AF (Apixaban versus Antiplatelet drugs or no antithrombotic drugs after anticoagulationassociated intraCerebral HaEmorrhage in patients with Atrial Fibrillation) is exploring the rate of vascular death or non-fatal stroke in patients given apixaban compared with antiplatelet therapy or no antithrombotics after sICH. ${ }^{58}$

> The optimal timing to restart antithrombotic therapy is unclear. Observational studies reported that avoiding anticoagulation for 7 days up to 10 weeks after sICH is safe. ${ }^{59,60}$.

\section{Conflicts of interest}

The authors have no conflicts of interest to declare.

\section{References}

1 van Asch CJ, Luitse MJ, Rinkel GJ et al. Incidence, case fatality, and functional outcome of intracerebral haemorrhage over time, according to age, sex, and ethnic origin: a systematic review and meta-analysis. Lancet Neurol 2010;9:167-76.

2 Demchuk AM, Dowlatshahi D, Rodriguez-Luna D et al. Prediction of haematoma growth and outcome in patients with intracerebral haemorrhage using the CT-angiography spot sign (PREDICT): a prospective observational study. Lancet Neurol 2012;11:307-14.

3 Sahni R, Weinberger J. Management of intracerebral hemorrhage. Vasc Health Risk Manag 2007;3:701-9.

4 Cordonnier C, Klijn CJ, van Beijnum J, Al-Shahi Salman R. Radiological investigation of spontaneous intracerebral hemorrhage: systematic review and trinational survey. Stroke 2010;41:685-90.

5 Wilson D, Adams ME, Robertson F, Murphy M, Werring DJ. Investigating intracerebral haemorrhage. BMJ 2015;350:h2484.

6 Stroke Unit Trialists' Collaboration. Organised inpatient (stroke unit) care for stroke. Cochrane Database Syst Rev 2013;(9):CD000197.

7 Brott T, Broderick J, Kothari R et al. Early hemorrhage growth in patients with intracerebral hemorrhage. Stroke 1997;28:1-5.

8 Balami JS, Buchan AM. Complications of intracerebral haemorrhage. Lancet Neurol 2012;11:101-18.

9 Parry-Jones AR, Paley L, Bray BD et al. Care-limiting decisions in acute stroke and association with survival: analyses of UK national quality register data. Int J Stroke 2016;11:321-31.

10 Morgenstern LB, Zahuranec DB, Sanchez BN et al. Full medical support for intracerebral hemorrhage. Neurology 2015;84:1739-44.

11 Ohwaki K, Yano E, Nagashima H et al. Blood pressure management in acute intracerebral hemorrhage: relationship between elevated blood pressure and hematoma enlargement. Stroke 2004;35:1364-7.
12 Anderson CS, Heeley E, Huang Y et al. Rapid blood-pressure lowering in patients with acute intracerebral hemorrhage. $N$ Engl J Med 2013;368:2355-65.

13 Qureshi AI, Palesch YY, Martin R et al. Effect of systolic blood pressure reduction on hematoma expansion, perihematomal edema, and 3-month outcome among patients with intracerebral hemorrhage: results from the antihypertensive treatment of acute cerebral hemorrhage study. Arch Neurol 2010;67:570-6.

14 Krishnan K, Scutt P, Woodhouse L et al. Glyceryl trinitrate for acute intracerebral hemorrhage: results from the Efficacy of Nitric Oxide in Stroke (ENOS) trial, a subgroup analysis. Stroke 2016;47:44-52.

15 Tsivgoulis G, Katsanos AH, Butcher KS et al. Intensive blood pressure reduction in acute intracerebral hemorrhage: a metaanalysis. Neurology 2014;83:1523-9.

16 Steiner T, Al-Shahi Salman R, Beer R et al. European Stroke Organisation (ESO) guidelines for the management of spontaneous intracerebral hemorrhage. Int J Stroke 2014;9:840-55.

17 Hemphill JC3rd, Greenberg SM, Anderson CS et al. Guidelines for the management of spontaneous intracerebral hemorrhage: a guideline for healthcare professionals from the American Heart Association/American Stroke Association. Stroke 2015;46:2032-60.

18 Intercollegiate Stroke Working Party. National clinical guideline for stroke, 5th edn. London: Royal College of Physicians, 2016.

19 Qureshi AI, Palesch YY, Barsan WG et al. Intensive blood-pressure lowering in patients with acute cerebral hemorrhage. $N$ Engl J Med 2016;375:1033-43.

20 Arima H, Tzourio C, Butcher K et al. Prior events predict cerebrovascular and coronary outcomes in the PROGRESS trial. Stroke 2006;37:1497-502.

21 Steiner T, Poli S, Griebe M et al. Fresh frozen plasma versus prothrombin complex concentrate in patients with intracranial haemorrhage related to vitamin $\mathrm{K}$ antagonists (INCH): a randomised trial. Lancet Neurol 2016;15:566-73.

22 Parry-Jones A. Cutting delays in reversing anticoagulation after intracerebral haemorrhage: three key changes at a UK comprehensive stroke centre. BMJ Qual Improv Rep 2015;4:u208763.w3521.

23 Pollack CVJr., Reilly PA, Eikelboom J et al. Idarucizumab for Dabigatran Reversal. N Engl J Med 2015;373:511-20.

24 Connolly SJ, Milling TJJ, Eikelboom JW et al. Andexanet alfa for acute major bleeding associated with factor Xa inhibitors. N Engl J Med 2016;375:1131-41.

25 Heidbuchel H, Verhamme P, Alings M et al. EHRA practical guide on the use of new oral anticoagulants in patients with non-valvular atrial fibrillation: executive summary. Eur Heart J 2013;34:2094106.

26 Baharoglu MI, Cordonnier C, Salman RA et al. Platelet transfusion versus standard care after acute stroke due to spontaneous cerebral haemorrhage associated with antiplatelet therapy (PATCH): a randomised, open-label, phase 3 trial. Lancet 2016;387:2605-13.

27 Al-Shahi Salman R. Haemostatic drug therapies for acute spontaneous intracerebral haemorrhage. Cochrane Database Syst Rev 2009;(4):CD005951.

28 Mayer SA, Brun NC, Begtrup K et al. Efficacy and safety of recombinant activated factor VII for acute intracerebral hemorrhage. N Engl J Med 2008;358:2127-37.

29 Meretoja A, Churilov L, Campbell BC et al. The spot sign and tranexamic acid on preventing ICH growth-AUStralasia Trial (STOP-AUST): protocol of a phase II randomized, placebocontrolled, double-blind, multicenter trial. Int J Stroke 2014;9:51924.

30 Sprigg N, Robson K, Bath P et al. Intravenous tranexamic acid for hyperacute primary intracerebral hemorrhage: protocol for a randomized, placebo-controlled trial. Int J Stroke 2016;11:683-94.

31 Kirollos RW, Tyagi AK, Ross SA, van Hille PT, Marks PV. Management of spontaneous cerebellar hematomas: a prospective treatment protocol. Neurosurgery 2001;49:1378-86. 
32 Mendelow AD, Gregson BA, Fernandes HM et al. Early surgery versus initial conservative treatment in patients with spontaneous supratentorial intracerebral haematomas in the International Surgical Trial in Intracerebral Haemorrhage (STICH): a randomised trial. Lancet 2005;365:387-97.

33 Mendelow AD, Gregson BA, Rowan EN et al. Early surgery versus initial conservative treatment in patients with spontaneous supratentorial lobar intracerebral haematomas (STICH II): a randomised trial. Lancet 2013;382:397-408.

34 Gregson BA, Broderick JP, Auer LM et al. Individual patient data subgroup meta-analysis of surgery for spontaneous supratentorial intracerebral hemorrhage. Stroke 2012;43:1496-504.

35 Morgenstern LB, Demchuk AM, Kim DH, Frankowski RF, Grotta JC. Rebleeding leads to poor outcome in ultra-early craniotomy for intracerebral hemorrhage. Neurology 2001;56:1294-9.

36 Takeuchi S, Wada K, Nagatani K, Otani N, Mori K. Decompressive hemicraniectomy for spontaneous intracerebral hemorrhage. Neurosurg Focus 2013;34:E5.

37 Adams RE, Diringer MN. Response to external ventricular drainage in spontaneous intracerebral hemorrhage with hydrocephalus. Neurology 1998;50:519-23.

38 Ziai WC, Tuhrim S, Lane K et al. A multicenter, randomized, double-blinded, placebo-controlled phase III study of Clot Lysis Evaluation of Accelerated Resolution of Intraventricular Hemorrhage (CLEAR III). Int J Stroke 2014;9:536-42.

39 Mould WA, Carhuapoma JR, Muschelli J et al. Minimally invasive surgery plus recombinant tissue-type plasminogen activator for intracerebral hemorrhage evacuation decreases perihematomal edema. Stroke 2013;44:627-34.

40 Hofmeijer J, Kappelle LJ, Klijn CJ. Antithrombotic treatment and intracerebral haemorrhage: between Scylla and Charybdis. Pract Neurol 2015;15:250-6.

41 Bailey RD, Hart RG, Benavente O, Pearce LA. Recurrent brain hemorrhage is more frequent than ischemic stroke after intracranial hemorrhage. Neurology 2001;56:773-7.

42 Biffi A, Anderson CD, Battey TW et al. Association between blood pressure control and risk of recurrent intracerebral hemorrhage. JAMA 2015;314:904-12.

43 Viswanathan A, Rakich SM, Engel C et al. Antiplatelet use after intracerebral hemorrhage. Neurology 2006;66:206-9.

44 Poon MT, Fonville AF, Al-Shahi Salman R. Long-term prognosis after intracerebral haemorrhage: systematic review and metaanalysis. J Neurol Neurosurg Psychiatry 2014;85:660-7.

45 Charidimou A, Kakar P, Fox Z, Werring DJ. Cerebral microbleeds and recurrent stroke risk: systematic review and meta-analysis of prospective ischemic stroke and transient ischemic attack cohorts. Stroke 2013;44:995-1001.

46 Vermeer SE, Algra A, Franke CL, Koudstaal PJ, Rinkel GJ. Long-term prognosis after recovery from primary intracerebral hemorrhage. Neurology 2002;59:205-9.

47 Gomez-Outes A, Terleira-Fernandez AI, Calvo-Rojas G, Suarez-Gea ML, Vargas-Castrillon E. Dabigatran, rivaroxaban, or apixaban versus warfarin in patients with nonvalvular atrial fibrillation: a systematic review and meta-analysis of subgroups. Thrombosis 2013;2013:640723.
48 Cannegieter SC, Rosendaal FR, Wintzen AR et al. Optimal oral anticoagulant therapy in patients with mechanical heart valves. N Engl J Med 1995;333:11-7.

49 Iung B, Rodes-Cabau J. The optimal management of anti-thrombotic therapy after valve replacement: certainties and uncertainties. Eur Heart J 2014;35:2942-9.

50 Eikelboom JW, Connolly SJ, Brueckmann M et al. Dabigatran versus warfarin in patients with mechanical heart valves. $N$ Engl J Med 2013;369:1206-14.

51 Lip GY, Nieuwlaat R, Pisters R, Lane DA, Crijns HJ. Refining clinical risk stratification for predicting stroke and thromboembolism in atrial fibrillation using a novel risk factor-based approach: the euro heart survey on atrial fibrillation. Chest 2010;137:263-72.

52 Hart RG, Pearce LA, Aguilar MI. Meta-analysis: antithrombotic therapy to prevent stroke in patients who have nonvalvular atrial fibrillation. Ann Intern Med 2007;146:857-67.

53 Nielsen PB, Larsen TB, Skjoth F et al. Restarting Anticoagulant treatment after intracranial hemorrhage in patients with atrial fibrillation and the impact on recurrent stroke, mortality, and bleeding: a nationwide cohort study. Circulation 2015;132:517-25.

54 Eckman MH, Rosand J, Knudsen KA, Singer DE, Greenberg SM. Can patients be anticoagulated after intracerebral hemorrhage? A decision analysis. Stroke 2003;34:1710-6.

55 ISRCTN registry. ISRCTN71907627: REstart or STop Antithrombotics Randomised Trial. www.isrctn.com/ISRCTN71907627 [Accessed 2 February 2017].

56 Horstmann S, Zugck C, Krumsdorf U et al. Left atrial appendage occlusion in atrial fibrillation after intracranial hemorrhage. Neurology 2014;82:135-8.

57 Reddy VY, Doshi SK, Sievert H et al. Percutaneous left atrial appendage closure for stroke prophylaxis in patients with atrial fibrillation: 2.3-year follow-up of the PROTECT AF (Watchman Left Atrial Appendage System for Embolic Protection in Patients with Atrial Fibrillation) trial. Circulation 2013;127:720-9.

58 van Nieuwenhuizen KM, van der Worp HB, Algra A et al. Apixaban versus Antiplatelet drugs or no antithrombotic drugs after anticoagulation-associated intraCerebral HaEmorrhage in patients with Atrial Fibrillation (APACHE-AF): study protocol for a randomised controlled trial. Trials 2015;16:393.

59 Phan TG, Koh M, Wijdicks EF. Safety of discontinuation of anticoagulation in patients with intracranial hemorrhage at high thromboembolic risk. Arch Neurol 2000;57:1710-3.

60 Majeed A, Kim YK, Roberts RS, Holmstrom M, Schulman S. Optimal timing of resumption of warfarin after intracranial hemorrhage. Stroke 2010;41:2860-6.

Address for correspondence: Dr Nikola Sprigg, Stroke, Division of Neuroscience, University of Nottingham, Clinical Sciences Building, City Hospital Campus, Nottingham NG5 1PB, UK. Email: nikola.sprigg@nottingham.ac.uk 